\begin{tabular}{|c|c|c|c|}
\hline$\bigcup_{\text {INESEG }}^{\text {INTERNATIONAL }}$ & $\begin{array}{r}\text { International } \\
\text { Res } \\
w w\end{array}$ & $\begin{array}{l}\text { ournal of Health Services } \\
\text { arch and Policy } \\
\text { lergipark.org.tr/ijhsrp }\end{array}$ & \\
\hline $\begin{array}{l}\text { SCIENCE AND } \\
\text { EDUCATION GROUP }\end{array}$ & e-ISSN:2602-3482 & DOI: $10.33457 /$ ijhsrp.670942 & IJHSRP \\
\hline
\end{tabular}

Review Article

\title{
CANCER, SIDE EFFECTS OF CHEMOTHERAPY AND NURSING CARE
}

\section{Çiçek SARUHAN}

Dicle University Ataturk School of Health, Nursing Department, Diyarbakır/TURKEY

Corresponding author:ciceksaruhan82@gmail.com

\begin{abstract}
Cancer is a complex disease that emerges from uncontrolled cell growth as a result of genetic and environmental factors. Although there are various types of cancer and standard procedures are applied to specific types of cancers, it is also a personal disease. Cancer treatment varies depending on the cancer type, placement, stage, general health of the individual and other factors. Furthermore, cancer treatment is complex, costly and requires a long time. As the prevalence of cancer increased in societies, the application of systemic chemotherapy and the occurrence of associated side effects also increased. Early monitoring and assessment have become important for early diagnosis of side effect symptoms and reduction and prevention of complications through symptom control. Social environments and families should be taken into consideration during patient assessment. Monitoring and assessment of cancer patients by all healthcare professionals and nurses during chemotherapy are at least as important as the follow-up of the disease. In this review, we aim to assess the factors leading to cancer, chemotherapy used to treat cancer and side effects associated with chemotherapy and to examine the applicable nursing care practices.
\end{abstract}

Keywords: Cancer, chemotherapy, side effects, nursing care

Received: January 6, $2020 \quad$ Accepted: March 16, 2020

\section{Introduction}

Cancer is a complex disease that emerges from uncontrolled cell growth as a result of genetic and environmental factors [1]. Cancer treatment varies depending on the cancer type, placement, stage, general health of the individual and other factors and cancer treatment is complex, costly and requires a long time [2].

\section{Epidemiology of Cancer}

'Cancer' actually refers to a group of diseases, all of which result from the uncontrolled abnormal growth of cells [3]. Its prevalence varies depending on age, gender, race, and region of residence. Cancer is the second leading cause of death in our country and in the world [4]. Cancer incidence and mortality rates for 2018 by sex and for 18 age groups $(0-4,5-9, \ldots, 79,80-84,85$ and over) were estimated for the 185 countries or territories of the world having a total population greater than 150,000 in 2018 [5]. It accounts for one-sixth of deaths in the world and one-fifth of deaths in our country [6]. According to the GLOBOCAN 2018 updates, 18.1 million new cancer cases and 9.6 million cancer deaths occurred in 
2018 worldwide, and, on average, there is about a $20 \%$ risk of getting cancer before age 75 , and $10 \%$ of dying from it [5]. It is estimated that a total of 19.3 million new cases of cancer will occur by 2025 [7]. This increase can be stopped only by adopting a healthy lifestyle behaviour and implementing evidencebased prevention strategies [6].

Cancer may develop as a result of many factors such as environmental exposure, lifestyle, and genetics. These include smoking and alcohol consumption, infections, reproductive and hormonal factors, dietary habits and physical inactivity, occupational exposure, radiation, water, and soil pollution in the residential areas, medicinal drugs, and naturally occurring chemical carcinogens.

\subsection{Smoking Consumption}

Smoking is a major public health problem [8]. Every year, 5.4 million people around the world die of lung cancer, heart diseases and other diseases caused by smoking [9]. Tobacco use is decreasing in high-income countries while it is high and increasing in most of the low and middle-income countries [8]. Tobacco smoke contains more than 7000 chemical substances, most of which are known to be carcinogenic. The chemicals in tobacco smoke contribute to the formation of cancer in a number of ways [9]. Smoking is responsible for the occurrence of $94 \%$ of lung cancer cases. Smokers have 24-36 times higher risk of lung cancer than non-smokers. Factors such as the duration of smoking, the number of cigarettes smoked, the age of smoking initiation affect the formation of cancer [10].

\subsection{Alcohol Consumption}

Alcohol use causes such cancer types as oral cavity, pharyngeal, laryngeal, esophageal, liver, colon, rectal and breast cancer. It is known that the consumption of alcoholic drinks increases the risk of the formation of many cancer types [11].

\subsection{Infections}

In the last thirty years, a correlation has been found between a number of chronic infections and the formation of cancer in cancer etiology studies [12]. Table 1 shows the carcinogenic agents that cause cancer in humans and the major cancer regions associated with these agents.

\subsection{Reproductive and Hormonal Factors}

It is reported that reproductive and hormonal factors play a significant role in a great number of cancer etiologies in women. These factors significantly affect the formation of breast, endometrial and ovarian cancers in particular [13-15]. Although hormonal factors may have an impact on a number of cancer types in men, they are described as less likely to be correlated with cancer [13, 16].

\subsection{Dietary Habits and Physical Inactivity}

It is reported that dietary habits and physical inactivity increase the rate of cancer formation and the most important effect of physical inactivity on cancer formation is weight gain and obesity. Further studies are needed to understand the impact of obesity in cancer types on survival. The diagnosis stage should include a careful examination explaining the relationship between phases and the treatment details [17, 18]. Nutrients, for the metabolic requirements of the organism, provide the necessary substances such as proteins, in addition to positive on our health they also contain ingredients such as secondary metabolites with effects [19]. 
Tab. 1. Carcinogenic agents causing cancer in humans and main cancer sites associated with these agents [12].

\begin{tabular}{|c|c|}
\hline Cancer site & Well-established human carcinogenic agents \\
\hline Stomach & Helicobacter pylori \\
\hline Liver & $\begin{array}{l}\text { Hepatitis B virus } \\
\text { Hepatitis C virus } \\
\text { Opisthorchisviverrini } \\
\text { Clonorchissinensis }\end{array}$ \\
\hline Cervix & Human papillomavirus, with or without HIV \\
\hline $\begin{array}{l}\text { Anogenital (penis, vulva, } \\
\text { vagina, anus) }\end{array}$ & Human papillomavirus, with or without HIV \\
\hline Nasopharynx & Epstein-Barr virus \\
\hline Oropharynx & $\begin{array}{l}\text { Human papillomavirus, with or without tobacco use/alcohol } \\
\text { consumption }\end{array}$ \\
\hline Non-Hodgkin lymphoma & $\begin{array}{l}\text { Helicobacter pylori } \\
\text { Epstein-Barr virus, with or without HIV } \\
\text { Hepatitis C virus } \\
\text { Human T-cell lymphotropic virus type } 1\end{array}$ \\
\hline Kaposi sarcoma & Kaposi sarcoma herpesvirus, with or without HIV \\
\hline Hodgkin lymphoma & Epstein-Barr virus, with or without HIV \\
\hline Bladder & Schistosomahaematobium \\
\hline
\end{tabular}

\subsection{Occupational Exposure, Radiation, Residential Area}

Exposure plays a significant role in cancers related to workplaces. For instance, asbestos, heavy metals, diesel motor emissions, polycyclic aromatic hydrocarbons, silica are well-known carcinogens in workplace exposures [20,21].

The dose of radiation exposure and the duration of exposure, the type of ray, the affected tissue, the sensitivity of organ and the age of affected person determine the severity of the potential disease $[22,23]$.

Environmental factors include everything non-genetic. For example, exposure to soil, ground and surface waters contaminated with chemicals and air, water and food in that residential area is a result of environmental pollution in all communities [24].

\subsection{Medicinal Drugs and Naturally Occurring Chemical Carcinogens}

Drugs are chemical substances used in medicine with the capacity to treat, prevent or alleviate the disease. Oral contraceptives, hormone replacement therapies, and anti-estrogens are drugs with carcinogenic potential [25].

Plants, fungi, lichens and some bacteria are natural chemicals that have unique pharmacological effects. Humans are exposed to most of these natural chemicals through water and food [26]. 


\section{Cancer Treatment}

Chemotherapy, radiotherapy, and surgery are the most widely used methods to treat cancer. In addition, different treatment approaches such as hormone therapy and biological methods can be used alone or as a complementary treatment with other methods [1]. Cancer treatment varies depending on the type, placement, and stage of cancer and the individual's general health and other factors [2]. The purpose of modern medicine is to prevent cancer. As the prevalence of cancer increases, its social and financial burden also increases [1].

\subsection{Chemotherapy in Cancer}

Despite the advancements in cancer treatment, chemotherapy is one of the common methods used alone or with other treatment methods [27]. However, chemotherapy may damage healthy cells while preventing the growth and proliferation of cancerous cells, which is known as side effects associated with chemotherapy [28, 29]. Cancer progression, treatment, and treatment toxicity have significant local and systemic effects (intestinal and oral mucosal epithelium, hematopoietic cells in bone marrow, hair follicle cells, etc.) on all systems [30].

\section{Side Effects of Chemotherapy and Nursing Care}

Oral Mucositis: It is a side effect identified in the late 1980s, developing in about $40-50 \%$ of patients treated with chemotherapy, manifesting itself with the inflammation of oral mucosa and accepted as a sign of leukopenia. The term "oral mucositis" or "buccalmucositis" is used in the international literature [31]. The oral cavity is sensitive to the direct and indirect toxic effects of chemotherapy. This is induced by a number of factors including high cell-turnover rate of oral mucosa, complex and diverse microflora of oral cavity and oral tissue trauma during normal oral function [27]. Mucositis may manifest itself as erythema, edema or ulceration which can be accompanied by effects ranging from a mild burning sensation to large and painful ulcers that worsen the patient's quality of life and limit basic oral functions such as speech, swallowing of saliva or eating. Its maximum expression is observed 7-10 days after chemotherapy and erythema progresses toward ulceration. This is a period when the patient feels great pain and discomfort and requires the administration of opioids and changes in diet in most cases. Then, mucositis gradually regresses 2-3 weeks after the administration of treatment, without leaving any scars, provided that the patient does not develop bone marrow suppression [31].

The main objective of mucositis treatment is to determine the risk factors and take preventive measures. Age and gender, genetic factors, poor oral hygiene, acute or chronic periodontal disorders, infections, malnutrition, therapy-induced dryness of mouth, dysfunctional salivation, suppressed immune system and smoking-alcohol consumption are patient-related risk factors that influence the development of mucositis [32]. The purpose of care is to mitigate the effect of oral microbial flora and prevent the development of opportunistic infections. It is recommended to avoid hot or cold foods, sour or spicy foods, fizzy drinks, alcohol and smoking which may irritate mucosa [28]. Following the formation of mucositis; oral care should be continued, antiseptic and analgesic mouthwashes should be used and, in the case of severe pain, narcotic analgesics should be administered. Also, nutritional support must be given to patients with undernutrition resulting from mucositis [32]. Close follow-up and 
assessment are highly important as weight loss, lack of appetite, cachexia, and dehydration may develop during the care of patients with mucositis. Patients should be evaluated within the week following the administration of chemotherapy and patients and families should be informed on the results of this evaluation [33, 34].

Nausea-Vomiting and Lack of Appetite: All fundamental factors that cause vomiting were explained in the early 1950s. Nausea-vomiting induced by chemotherapy is related to a significant reduction in quality of life, which is perceived by patients as a negative effect of the therapy [35, 36]. Nausea-vomiting induced by chemotherapy, the most commonly reported side effect, is observed in 38-80\% of patients receiving cancer treatment and its severity varies from patient to patient [37]. Lack of appetite, on the other hand, refers to weight loss as a result of less intake of nutrients than an individual's metabolic needs. Lack of appetite and irregular and insufficient intake of food are among the most challenging side effects of cancer observed in about 54\% of cancer patients [38]. Uncontrollable nausea and vomiting has negative effects not only on fluid and electrolyte imbalance, dehydration, anorexia, weight loss, decreased absorption and renal excretion of the drug but also on the social life, work-life, daily life activities and psychological state of the individual [33]. During patient care; antiemetic drugs should be administered following the administration of chemotherapeutic drugs and at least half an hour before each meal, fluid and well-tolerated soft foods should be provided to reduce nausea, body weight, fluids taken in and discharged out of the body and electrolyte values should be monitored, patients should be told that breathing through the mouth is better during nausea and vomiting, and relaxation techniques should be used before and during the therapy [33, 38].

Pain: Pain is one of the most common and challenging symptoms in cancer patients. Pain may result not only from cancer but the therapy may also lead to chronic pain [39]. 1/4 of diagnosed cancer patients, $1 / 3$ of patients under ongoing treatment and 3/4 of advanced-stage patients experience pain [40]. Pain, as defined by the International Association for the Study of Pain (IASP), is "an unpleasant sensory and emotional experience associated with actual or potential tissue damage, or described in terms of such damage." [41]. Pain may cause increased discomfort, anxiety and depression, insomnia, fatigue, lack of appetite in patients and may have a severe effect on the patient's self-care skills, daily activities, and communication with family and friends [40, 42]. Since pain is subjective, "Analgesic Ladder" is frequently applied as a therapy algorithm as part of the Cancer Pain Relief program recommended by the World Health Organization [41]. In ladder therapy, the aim is to choose a step based on the severity of pain, prefer oral administration initially, and use agents that will take the pain under control throughout the day, ensure that the treatment is personal and pay attention to details. Mostly, non-opioid analgesics such as paracetamol and non-steroidal anti-inflammatory drugs (NSAID) alone or in combination are used in the first step and if this fails to relieve the pain, the next step is applied. In the second step, a mild or mild-to-moderate analgesic such as codeine is added to the treatment described in the first step. If the pain continues, the third step is applied and the mild opioid is replaced with a strong opioid such as morphine and patients are titrated to a pain-relieving dose in order to prevent the formation of pain [43, 44]. Pain management requires an evaluation with a multidimensional and multidisciplinary approach and then the use of pharmacological and nonpharmacological therapeutic strategies [42]. Because of the effect of pain on the patient's physical and psychological symptoms, functionality and comfort should be evaluated and the patient's false information and fears, if any, should be eliminated [33, 38]. 
Bleeding Risk: Bleeding occurs as a result of the reduced capacity of platelet production due to the bone marrow suppression caused by chemotherapy drugs [38]. The most common cause of bleeding is a reduction in the number of circulating platelets below $100,000 \mathrm{~mm}^{3}$ and the most common bleeding sites are skin, mucous membranes, intestines, brain, urinary and respiratory tracts [33]. Patient should be monitored for bleeding signs, necessary precautions should be taken against the risk of reduction, patient and relatives should be informed on the importance of maintaining skin and mucous membrane integrity, soft toothbrushes should be used in dental care, electric shaver should be used, no invasive procedure should be performed unless necessary, non-adhesive humid wound adhesive products should be used in procedures and clothing that prevent trauma and irritation should be recommended $[33,38]$.

Infection Risk: An infection occurs when a microorganism enters the human or animal body in some fashion, settles and multiplies there. Organisms that cause disease are usually viruses, bacteria, rickettsia and fungi which are also the agents that often lead to infection in cancer patients. Methods applied to treat cancer patients and occasionally the disease itself cause immune insufficiency [45]. Some chemotherapy drugs damage the bone marrow functions, reduce the ability to generate new cells and the number of leukocytes decreases as a result of rapid cell death and, thus, the number of neutrophils decreases [33, 38]. Neutropenia occurs 7-14 days after the chemotherapy application [38]. A reduction in the count of neutrophils below $1000 \mathrm{~mm}^{3}$ increases the risk of infection [33]. Fever which is the most significant sign of infection is the most important and sometimes the only symptom of infection particularly in neutropenic patients [30]. During patient care; patients should stay in single rooms, if possible, and the number of visitors should be restricted, patients should be monitored for any sign of infection, skin integrity should be preserved, daily hygiene requirements should be fulfilled, aseptic techniques should be followed in all invasive procedures, daily leukocyte (especially the count of neutrophils) value should be monitored, the physician should be informed of suspicious circumstances and culture samples should be taken where necessary, precautions should be taken to prevent constipation, raw or undercooked meat, seafood, eggs, and insufficiently-washed fruit and vegetables should be avoided, patients should be helped with their deep breathing and coughing exercises, activities that are appropriate for their health status should be planned, flowers and plants should be avoided [33, 38, 45].

Constipation and Diarrhea: Constipation and diarrhea are not diseases; they are symptoms varying from person to person, described in different ways and generally developing secondarily to another disease. Constipation refers to experiencing one or more of the symptoms of hard feces, decrease in the number of defecation, need for intense straining when defecating, sensation of incomplete evacuation in bowels, insufficient defecation, pressure on the anus or near the vaginal area, insertion of fingers or spending long times in the toilet [46]. The prevalence of constipation has been reported to be $40-64 \%$ in patients with cancer receiving palliative care, and $70-100 \%$ in patients receiving cancer treatment [47]. Diarrhea is defined as abnormal, watery, lose defecation more than 34 times a day, whose quantity varies depending on age and dietary factors (more than 250-300 millimeters) [46]. Constipation and diarrhea develop in cancer patients due to many reasons [38]. In patient care, factors that cause both conditions should be determined and bowel habits should be assessed through regular monitoring. In constipation cases, a prophylactic bowel protocol can be initiated in patients with risk factors on doctor's advice. Rectal administration (using suppository, enema) should be avoided in neutropenic and thrombocytopenic patients and special attention should be given to hydration in patients suspected to have an intestinal obstruction. In diarrhea cases; its onset, frequency, content, 
and duration should be followed up and fluid-electrolyte losses, abdominal pain, debility and fever signs of patients should be evaluated, daily weight and vital signs of patients should be monitored and patients should be informed on emergencies (fever above $38^{\circ} \mathrm{C}$, bloody stool, acute abdominal pain and cramps, occurrence of distension, sensory loss, dizziness, debility, confusion, excessive thirst, dark urine, potential dehydration signs) $[38,46]$.

Disturbance of Sleep Pattern and Fatigue: Sleep is the most important indicator of overall health and well-being [48]. Insomnia is defined as difficulty in falling asleep, frequent night-time awakenings, early waking in the morning, having nightmares, difficulty in getting out of bed and may affect the problem-solving skills of individuals, hinder their positive coping strategies and impair their adaptation to new circumstances [49]. The frequency of sleep disorders in cancer patients is reported to be between 30-58\% [50]. Another symptom related to sleeplessness is fatigue. Fatigue is a problem that has a physical, emotional and cognitive impact on the individual and prevents them from carrying out daily life activities [51]. Reported fatigue rate of $80 \%$ for patients receiving chemotherapy [52]. Cancerinduced fatigue is a more serious problem compared to the fatigue experienced by healthy individuals. The patient does not feel relaxed after resting, which is one of the symptoms observed in cancer patients as a result of the disease and the treatment $[49,53]$. Fatigue is often associated with the type and quantity of the treatment administered, anemia, weakness in muscles, other concurrent diseases, insomnia, emotional problems and pain $[51,53]$. One of the most significant factors in this chapter is to identify insomnia and fatigue problems $[49,51]$. In insomnia management, patients and their relatives should be informed on the effect of treatments on the sleep pattern, importance of maintaining the sleep hygiene, management of symptoms that cause insomnia (such as pain, nausea, vomiting), use of body-mind practices that are effective in coping with insomnia (massage, reflexology, music therapy, art therapy, progressive muscle relaxation exercises, yoga, warm shower and tools to help with sleep (such as cool thin pillows used by breast cancer patients) and in which cases they should go to a health institution [49]. In the management of fatigue, the care should be planned by taking the patient's individual characteristics into consideration and the patient should be taught the necessity and importance of being active during the day, having a regular sleep pattern, consuming an adequate and balanced diet, learning to cope with stress and negative feelings using relaxation techniques, maintaining the social relationships and attending various activities and going to a health institution in challenging circumstances [51,53].

Alopecia: Chemotherapy affects both carcinogenic and non-carcinogenic cells. Alopecia occurs as a result of a change in hair follicles and although not life-threatening in chemotherapy patients, it affects their body image, self-confidence, family and social life and is one of the most common symptoms [53, 54]. Hair is an object associated with personal maturity and stages of life for many individuals and plays a major role in sexual life. Hair loss is associated with the aging process as well as the loss of sexuality, decreased personal attractiveness, being sick and dying. Socially, hair loss leads to the loss of sense of self, lower social status and destruction of personality and may cause such thoughts and feelings as anger, sadness, embarrassment, and fear of getting rejected [53]. Hair loss generally starts 2-3 weeks following chemotherapy and hair regrows within 8 weeks after the end of therapy [33]. Patients should be advised to cut their hair as short as possible and told that hair loss is a temporary side effect, their hair will regrow and only its structure might be different. Their thoughts and opinions about it should be received, they should be given the opportunity to express themselves and supported in 
controlling and dealing with signs such as sadness and hopelessness that may be felt due to the change in body image [53]. The use of wigs can be recommended [54].

Extravasation: It is a condition that occurs when the intravenously infused chemotherapy agent inadvertently leaks to the surrounding tissues rather than the intravenous space. Extravasation may occur with either a peripheral catheter or central venous tools and may lead to more injury [54]. Tissue injury due to extravasation is affected by such factors as the intensity of the chemotherapy agent, the amount of fluid leaked into the tissue, the duration of tissue's exposure to the drug and the site of extravasation. In addition to these factors, the patient's characteristics, as well as many other factors related to the clinical process, affect the development of extravasation [55]. Especially patients and their caregivers should be informed on monitoring and reporting the local pain, swelling, temperature increase, changes in the skin that may be observed on the intravenous administration site. Patients should be followed up by taking the chemotherapeutic agent's necrotizing capacity into consideration (if vesicant extravasation has developed, the site should be monitored for 24 hours at 8-hour intervals and the follow-up should continue for a week until the symptoms subside). Furthermore, the nurse should evaluate the patientinduced problems and the patient's anxiety level and plan the procedures. In addition, the chemotherapy follow-up should be recorded in the nurse observation chart and protocols for hot and cold applications after potential extravasation should be prepared and hung in places easily accessible to everyone. Leaflets explaining what can be done in the first 48 hours of extravasation and phone numbers to receive consultancy should be available in the therapy units. Nurses and healthcare professionals in charge of the treatment and management of cytotoxic agents should have up-to-date information to manage the risk of vesicant and irritant drugs. Standards should be developed and a risk management procedure should be organized for the management of vesicants [55]. Patients with extravasation should avoid wearing tight clothes, shoes, socks, belts, accessories and rough fabric and walking outside in open shoes or barefoot. Dressings should not be too tight, extremely adhesive bandages should not be used and patients should be trained to not use strong chemical detergents and bleaches, irritant detergents and bleaches and other chemical agents, utilize protective gloves if chemicals have to be used, protect their hands in physically demanding activities, avoid direct contact with vapour, protect the protruding bone sites against any frictions, use sunscreens before going out, consume at least 8-12 glasses of water daily per day unless there is a hindrance and eat food rich in protein, B and C vitamins [38].

Changes in Behaviours-Mood: Cancer is one of the leading health problems of our age. Cancer is perceived as a chronic disease that involves helplessness and ambiguity, brings to mind death in suffering and pain, arouses the feelings of guilt and anxiety, panic and chaos [56]. Although the survival rates of patients have increased thanks to the advancements in the diagnosis and treatment of cancer, physical and psychosocial disorders impair the adjustment mechanism of patients and have a negative impact on their expectations regarding the future [53, 56]. Psychological symptoms observed in patients treated with chemotherapy affect the individuals negatively and decrease their well-being [53]. Changes in behaviours and moods of cancer patients may result from a great number of factors including admission to the hospital, surgical intervention, progression of the disease, prolongation of the treatments, lack of social support, living alone, having conflicts with family members, financial problems, inadequate access to healthcare services, having a young/dependent child, having disease or treatment-induced symptoms. Such problems should be detected in the early stages and managed effectively before they become chronic [57]. It is highly important to determine how the cancer patients perceives their disease and the factors affecting this perception in order to mitigate the patient's 
psychological anxiety and pain, ensure their adaptation to the therapy, increase their quality of life, help them to express their feelings and support them in coping with the multidimensional crisis caused by the disease in a healthy manner [58]. Individuals should be supported to share their anxiety and fears about the disease and the future, they should express positive feelings about themselves, they should be given strength to cope with their illness and associated negative feelings, social support systems should be used, they should be encouraged to participate in motivating activities, the appropriate conditions should be established to help them to live their religious beliefs and share them whenever they wish [38, 56]. Since disagreement is present among patients, patient's relatives and doctors carrying out the treatment about informing the patients on their medical diagnosis, there are still ongoing discussions on this problem [58].

Sexual Problems: Sexuality is a concept with biological, psychological and sociological aspects that are affected by the cultural structure. Individual's perceptions of sexuality and sexual behaviours are influenced by family, society, value judgments, customs, and traditions [59]. Sexual health refers to the state of physical, psychosocial and sociocultural well-being in relation to sexuality, free expression of sexuality, having and maintaining an informed, safe and responsible sexual life based on mutual respect in sexual relations [60]. In cancer cases, the four important components of sexual health which are body image, gender role function, sexual functions, and reproductive ability may be impaired due to the disease and the treatment [61]. The side effects of cancer drugs may limit the sexual activity and cause such problems as reduced sexual desire, sexual arousal disorder, difficulty in orgasm, erectile dysfunction and ejaculation problems in men and dyspareunia associated with vaginal dryness in women [60]. All patients diagnosed with cancer should be informed about the changes in sexuality starting from the diagnosis until before the therapy is started. Sexuality is a highly sensitive subject that should be approached with a non-judgmental attitude in cancer patients. The main objective should be to increase the quality of life of the patient and his/her spouse. In order to help the patients, healthcare professionals should identify their own thoughts and feelings, values and beliefs regarding sexuality and be careful to not reflect their feelings toward the patients [59]. It should be remembered that each patient and their family is unique, a strong communication based on mutual trust should be established with the patients and their spouses, and they should be assured that the confidentiality and secrecy of their meetings and medical records will be maintained. Since the patient's culture, beliefs and values affect his or her sexuality and sexual functions, healthcare staff should be knowledgeable thereon, the history of sexual function should be obtained in a suitable environment after the approval of both spouses is received [38, $60]$.

\section{Conclusion}

In a detailed literature review, it has been observed that cancer patients generally do not have sufficient knowledge about their condition and are not actively engaged in their own care. Cancer patients are often faced with new needs that are beyond their skills and knowledge throughout the therapy process and thus need training and advice more than ever. Nurses should help patients to learn the healthcare practices so that they can regain and maintain their health, determine the patient's needs and arrange the care schedule to ensure that the patients can cope with their limitations and improve their existing skills, and have the patients engaged in their own care. The patients' self-care ability will contribute to their adaptation to the therapy. 
The compliance to the Research and Publication Ethics: This study was carried out in accordance with the rules of research and publication ethics.

Ethical Process: Ethics committee approval is not required for this study.

\section{References}

[1] Baykara, O.,"Current Modalities in Treatment of Cancer", Balıkesir Health Sciences Journal, 5, 154-65, 2016.

[2] Bakar, C.,"Epidemiology of Cancer in the World and Turkey", Turkey Clinics J Med Genet-Special Topics, 2, 49-59, 2017.

[3] Clare C.," Cancer", Principles of Applied Pathophysiology (Ed. M. Narrve I. Peate (Translation Ed. M. Y1lmaz and Z. Seki)), 1.Edition, İstanbul Medical Bookstore, 36-65, 2018.

[4] Can, G.,"Cancer", Pathophysiology (Ed. F. EtiAslan, N. Olgun), Academician Medical Bookstore, 33-51, 2017.

[5] Ferlay, J., Colombet, M., et al. "Estimating the Global Cancer Incidence and Mortality in 2018: GLOBOCAN Sources and Methods ", International Journal of Cancer, 144, 1941-1953, 2019.

[6] Hacıkamiloğlu, E., Gültekin, M. et al. Turkey Cancer Statistics (İ.Şencan, B. Keskinkılıç), T.C. Ministry of Health Public Health Agency of Turkey, Ankara, 2017.

[7] Saatç1, E.,"Cancer Epidemiology: Worldwide and Turkey", Turkey Clinics J Fam Med-Special Topics, 5, 1-8, 2014.

[8] Thun, J.M., Nilson, J.R., et al."The Global Tobacco Epidemic", World Cancer Report 2014 (Ed. B.W. Stewart, P.C. Wild ), 81-87, WHO, Lyon, France: 2014.

[9] Samet, J.M., Gupta P.C., Ray C.S.,"Tobacco Smoking and Smokeless Tobacco Use", World Cancer Report 2014 (Ed. B.W. Stewart, P.C. Wild ), 88-95, WHO, Lyon, France: 2014.

[10] Akkoçlu, A.,"Lung Cancer Epidemiology". (Ed. N.M. Mandel, T. Ece), National Lung Congress Book, Turkish Lung Cancer Association, Nevşehir, Kapadokya, 1-2, 2013.

[11] Rehm, J., Shield, K.,"Alcohol Consumption", World Cancer Report 2014 (Ed. B.W. Stewart, P.C. Wild), 96-104, WHO, Lyon, France: 2014.

[12] Franceschi, S., Herrero, R.," İnfections", World Cancer Report 2014 (Ed. B.W. Stewart, P.C. Wild), 105-114, WHO, Lyon, France: 2014.

[13] Brinton, LA.," Reproductive and Hormonal Factors", World Cancer Report 2014 (Ed. B.W. Stewart, P.C. Wild ), 116-123, WHO, Lyon, France: 2014.

[14] Pinar, G., Algier, L., Kaya N.,'Determination of the Risk Factors in Individuals with Gynecological Cancer", International Journal of Hematology and Oncology, 4, 208-216, 2008.

[15] Eroğlu, K., Koç, G.,"Gynacological Cancer Control and Nursing", Journal of Hacettepe University Faculty of Nursing, 1, 77-90, 2014.

[16] Çelik, S., Bozkurt, Ö., et al.," Association Between Hormonal Evaluation Before Prostate Neddle Biopsy and Locally Advanced Prostate Cancer", Bulten in Urooncology, 15, 52-56, 2016. 
[17] Atabilen, B., Akbulut, G.," Different Mechanisms and the Role Nutrition in the Formation of Colorectal Cancer", Turkey Clinics J Med Sci, 39, 89-107, 2019.

[18] Willett, W.C., Key, T., Romieu, I.," Diet, Obesity and Physical Activity", World Cancer Report 2014 (Ed. B.W. Stewart, P.C. Wild), 124-133, WHO, Lyon, France: 2014.

[19] Ar1, M., Öğüt, S., Kaçar Döğer F.,"Role of Antioxidants in Cancer Prevention", Adnan Menderes University Journal of Health Sciences, 2, 67-74, 2017.

[20] Siemiatycki, J.," Occupation", World Cancer Report 2014 (Ed. B.W. Stewart, P.C. Wild), 134150, WHO, Lyon, France: 2014.

[21] Sandal, A., Kılıçkap, S., Yıldız A.N.,"Overviewof OccupationalCancer", Turkish Medical Association Journal of Occupational Health and Safety, Temmuz-Ağustos-Eylül, 17, 41-47, 2017.

[22] Arslan, N.,"The Effects of Radiation on Biological Systems ", Nucl Med Semin, 3, 178-183, 2017.

[23] Seven Uzun, A.,"The Negative Effects of Natural and Unnatural Radiation Sources which are being Exposed to in Daily Life on Students' Life Qualities and Protection Recommendations", The Journal of Kesit Academy, 2, 322-335, 2016.

[24] Cohen, A.J., Cantor, K.P.,"Pollution of Air, Water and Soil", World Cancer Report 2014 (Ed. B.W. Stewart, P.C. Wild ), 151-160, WHO, Lyon, France: 2014.

[25] Friis, S., Olsen, J.H.," Pharmaceutical Drugs ", World Cancer Report 2014 (Ed. B.W. Stewart, P.C. Wild), 161-168, WHO, Lyon, France: 2014.

[26] Riley, R.T.,"Naturally Occurring Chemical Carcinogens ", World Cancer Report 2014 (Ed. B.W. Stewart, P.C. Wild ), 169-176, WHO, Lyon, France: 2014.

[27] Lopez, B.C.,"Oral Toxicity Produced by Chemotherapy: A Systametic Review", Journal of Clinical Experimental Dentistry, 6, 81-90, 2014.

[28] Çıtlak, K., Kapucu S.,"Current Approaches to Oral Mucositis Prevention and Treatment in Patients Receiving Chemotheraphy: Evidence Based Practices ", Journal of Hacettepe University Faculty of Nursing, 2, 70-75, 2015.

[29] Gür Ceylan, S., 'The Analysis of Caregiving Burden and Life Quality of Caregivers Who Care for Cancered Patients , Master Thesis, Harran University, Şanlıurfa, Turkey,2018.

[30] Hintistan, S., Çilingir, D., et al.," Applications of Patients with Hematologic Cancers for Experienced Symptoms due to Chemotherapy", Gümüşhane University Journal of Health Sciences, 1, 153-164, 2012.

[31] Lopez, B.C., Bagan Sebastian, J.V.,"Treatment of Oral Mucositis due to Chemotherapy ", Journal of Clinical Experimental Dentistry, 8, 201-209, 2016.

[32] Çubukçu, N.Ü., Çınar, S.,"Can Oral Mucositis be Prevented in Patients Receiving Chemotherapy?", Journal of Marmara University Institute of Health Sciences, 2, 155-163, 2012. 
[33] Eti Aslan, F., Olgun, N., Uzun, Ö." Oncology", Care in Internal and Surgical Diseases (A. Karadakovan, F. Eti Aslan), 4. Edition, 1.Volume, Academician Medical Bookstore, 185-211, 2017.

[34] Güngörmüş, Z., Erdem Bulut, Ö.," Oral Mucosıtıs and Life Qualıty in Cancer Patıents ", Acıbadem University Journal of Health Sciences, 5, 24-30, 2014.

[35] Navari R.M.," Introduction." Management of Chemotheraphy Induced Nausea and Vomiting (Ed. M.N. Rudolp), 1-4, Adis, 2016.

[36] Andrews P.L.R., Rudd J.A.," The Physiology and Pharmacology of Nausea and Vomiting Induced by Anticancer Chemotherapy in Humans ", Management of Chemotherapy-Induced Nausea and Vomiting (Ed. M.N. Rudolp), 5-44, Adis, 2016.

[37] Arslan, M., Özdemir, L.," Complementary and Alternative Theraphy Methods Used in the Management of Chemotheraphy Induced Nausea and Vomiting ", Turkish Journal of Oncology, 30, 82-89, 2015.

[38] Uğur, Ö.", Symptom Management of Cancer Patients ", Turkish Journal of Oncology, 29, 125135, 2014.

[39] Bennett, M.I., Stein, K., et al.,"The IASP Classification of Chronic Pain for ICD-11: Chronic Cancer Related Pain", Pain, 160, 38-44, 2019.

[40] Yu, Y., Zhou, J., et al."The Preliminary Application of Assessment System for Cancer Pain Management", European Review for Medical and Pharmacological Sciences, 19, 1164-1169, 2015.

[41] Arslan, M., Albaş, S., et al., "The Evaluation of the Effectiveness of Palliative Pain Management in Cancer Patients with Visual Analogue Scale ", Fam Pract Palliat Care, 1, 5-8, 2016.

[42] O’Brien, T., Kane C.M.,"Pain Services and Palliative Medicine an Integrated Approach to Pain Management in the Cancer Patient", British Journal of Pain, 8, 163-171, 2014.

[43] Arslan, D., Tatl1, A.M., Üyetürk, Ü., "Cancer Related Pain and Treatment ", Abant Medikal Journal, 2, 256-260, 2013.

[44] Aydoğan, F., Uygun, K.,"Cancer Patients Palliative Treatments ", Clinical Development, 24, 4-9, 2011.

[45] Kapucu, S.," Infection in Cancer Patients Receiving Palliative Care and Infection Management, ACU Journal of Health Sciences, 9, 366-370, 2018.

[46] Ören, B.,"Constipation and Diarrhea in Intensive Care Patients ",Journal of Intensive Care Nursing, 20, 37-43, 2016.

[47] Ünsar, S., Özdemir, Ö.,"Constipation", Oncology Nursing (Ed. G. Can), Nobel Medical Bookstores, 463-472, 2020.

[48] Bilsin, E., Özalp Gerçeker G.,"Sleep Disorders in Childhood Cancers ", Dokuz Eylul University Faculty of Nursing Electronics Journal, 11, 61-64, 2018. 
[49] Baykal, D., Can, G., et al.,"Sleep Problems", Evidence Based Palliative Care in Cancer Patient (Ed. G. Can), Nobel Medical Bookstores, 125-139, 2017.

[50] Baykal, D.,"İnsomnia", Oncology Nursing (Ed. G. Can), Nobel Medical Bookstores, 359-366, 2020.

[51] Baykal, D., Can G., et al.,"Fatigue," Evidence Based Palliative Care in Cancer Patient (Ed. G. Can), Nobel Medical Bookstores, 103-124, 2017.

[52] Usta Yeşilbakan, Ö."Fatigue ", Oncology Nursing (Ed. G. Can), Nobel Medical Bookstores, 347$358,2020$.

[53] Doğan, I.,"Symptom Control and Satisfaction with Nursing Care in Patients Receiving Chemotherapy", T.C. Trakya University Institute of Health Sciences Nursing Graduate Program, Master's Thesis, 2019.

[54] Roe, H., Lennan, E.,"Role of Nurses in the Assesment and Management of ChemotherapyRelated Side Effects in Cancer Patients", Nursing: Research and Reviews, 4, 103-115, 2014.

[55] Arslan, D., Aysever U., et al.," Incidence and Causes of Extravasation in Patients Admitted to Chemotherapy Treatment Centers for Medication Therapy ", Dokuz Eylul University Faculty of Nursing Electronics Journal, 11, 113-119, 2018.

[56] Kavradım Turan, S., Özer Canl1, Z.,"Hope in Patients with Cancer ", Current Approaches in Psychiatry, 6, 154-164, 2014.

[57] Kaya, F., Daştan Bostancı, N., Buzlu, S.," Distress". Evidence Based Palliative Care in Cancer Patient (Ed. G. Can), Nobel Medical Bookstores, 157-175, 2017.

[58] Karabulutlu Yılmaz, E., Karaman, S.,"Evaluation of Cancer Patients Perception of Illness ", HSP, 2, 271-284, 2015.

[59] Temiz, G.," Chemotherapy Applied Evaluation of Quality of Life and Health Care Requirements in Patients with Cancer", T.C. Halic University Health Sciences Institute Doctorate Thesis, 2016.

[60] Coşkun, A., Özerdoğan, N., "Sexual Health inPalliatıve Care", ACU Journal of Health Sciences, 9, 371-377, 2018.

[61] Geyikçi, R., Çakmak, S., et al.," Correlation of Anxiety and Depression Levels with Attitudes towards Coping with Illness and Sociodemographic Characteristics in Patients with A Diagnosis of Breast Cancer", Dusunen Adam The Journal of Psychiatry and Neurological Sciences, 31, 246$257,2018$. 